\title{
COMO A IMPLEMENTAÇÃO DAS BOAS PRÁTICAS DE FABRICAÇÃO (BPF) AUXILIAM A COMPETITIVIDADE E A QUALIDADE EM UMA INDÚSTRIA
}

\section{HOW THE IMPLEMENTATION OF GOOD MANUFACTURING PRACTICES (GMP) ASSIST COMPETITIVENESS AND QUALITY IN AN INDUSTRY}

\author{
David Vinicíos Chiarello Buzinaro - davidbuzinaro@gmail.com.br \\ Angelita Moutin Segoria Gasparotto - angelita.gaspaotto@ fatectq.edu.br \\ Faculdade de Tecnologia de Taquaritinga (FATEC) -SP - Brasil
}

DOI: 10.31510/infa.v16i2.662

\section{RESUMO}

As empresas estão buscando a implantação das Boas Práticas de Fabricação (BPF) devido à competitividade, melhoria contínua, exigência do mercado consumidor e, principalmente, a higiene alimentar dos seus produtos. Os sistemas de gestão, controle de qualidade e treinamentos dos colaboradores são, atualmente, um diferencial na indústria alimentícia. A qualidade é a principal característica das indústrias. Esse fato se dá devido à mudança de postura dos consumidores mundialmente, os quais procuram produtos que tragam e transmitam segurança, sejam atraentes e, além disso, sejam fabricados de forma sustentável. Nesse contexto, existe uma forma para que se possa obter e garantir a qualidade e a garantia nas indústrias: a implementação do programa de Boas Práticas de Fabricação - BPF's.

Palavras-chave: BPF. GMP. Gerenciamento. Indústria. Produção.

\begin{abstract}
Companies are seeking the implementation of Good Manufacturing Practices (GMP) due to competitiveness, continuous improvement, demand from the consumer market and, mainly, the food hygiene of their products. Employee management, quality control and training systems are currently a differential in the food industry. Quality is the main feature of industries. This is due to the changing attitudes of consumers worldwide, who are looking for products that bring and convey safety, are attractive and, in addition, are manufactured sustainably. In this context, there is a way to obtain and guarantee quality and assurance in industries: the implementation of the Good Manufacturing Practices (GMP) program.
\end{abstract}

Keywords: BPF. GMP. Management. Industry. Production. 


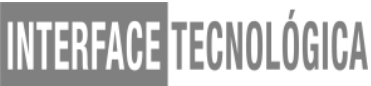

\section{INTRODUÇÃO}

De acordo com Marchiori (2015), as empresas estão buscando a implantação das Boas Práticas de Fabricação (BPF) devido à competitividade, melhoria contínua, exigência do mercado consumidor e, principalmente, a segurança alimentar dos seus produtos. Os sistemas de gestão, controle de qualidade e treinamentos dos colaboradores são, atualmente, um diferencial na indústria alimentícia.

As Boas Práticas de Fabricação têm como objetivo evitar a contaminação dos produtos. Elas abrangem desde a recepção das matérias-primas até o produto final, sendo que, na legislação brasileira, as BPF são obrigatórias para todos os estabelecimentos produtores e indústrias, sendo que, para avaliar o nível de implantação das Boas Práticas de Fabricação, é utilizada a lista de verificação (check list) presente na $\operatorname{RDC~}{ }^{\circ} 275$, de 21 de outubro de 2002 da Agência Nacional de Vigilância Sanitária (ANVISA). Por meio dos estudos realizados, observam-se os itens não conformes e buscam-se ações corretivas para a adequação dos requisitos, eliminando ou reduzindo os riscos que possam comprometer a saúde do consumidor (GENTA, 2005).

Segundo Silva (2011), a qualidade é a principal característica nas indústrias. Esse fato se dá devido à mudança de postura dos consumidores mundialmente, os quais procuram produtos que tragam e transmitam segurança, sejam atraentes e, além disso, sejam fabricados de forma sustentável. Nesse contexto, existe uma forma para que se possa obter e garantir a qualidade e a segurança nas indústrias: a implementação do programa de Boas Práticas de Fabricação BPF's. As BPF's são procedimentos que devem ser adotados pelas indústrias com o objetivo de garantir a qualidade higiênico-sanitária de acordo com a legislação vigente.

Para Veronezi (2015), dentre os sistemas utilizados para a implantação da qualidade, uma das ferramentas para se obter padrão de qualidade da produção é a adoção das Boas Práticas de Fabricação (BPF), que formam a base da gestão da segurança e da qualidade de uma indústria. As Boas Práticas de Fabricação abrangem um conjunto de princípios e regras que devem ser adotados pelas indústrias com o propósito de assegurar a qualidade higiênico-sanitária e a conformidade dos gêneros produzidos conforme a legislação vigente. 


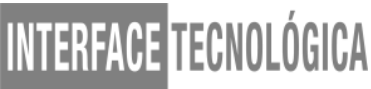

O presente artigo apresenta, primeiramente, a fundamentação teórica sobre a evolução da qualidade e a implantação das BPF em indústrias de alimentos. Em seguida, nos resultados e discussões é apresentado como as BPF auxiliam na gestão da qualidade na indústria alimentícia e sua respectiva implantação.

\section{FUNDAMENTAÇÃO TEÓRICA}

Pode-se dizer que a preocupação com a qualidade do produto tem origem nos primórdios da produção em escala, no início do século XIX, com a utilização de medidas, padrões e gabaritos na indústria bélica americana. Um século depois, houve a introdução da administração científica, com Taylor (1911); anos mais tarde, teve início o controle estatístico com Shewhart (1931), e, na década de 1950, o surgimento de conceitos como Controle Total da Qualidade, com Armand Feigenbaum (1956), e Custos da Qualidade e Não Qualidade, com Joseph Juran (1951) (VERONEZI, 2015).

Segundo Silva (2011), a qualidade evoluiu da adequação ao padrão para a adequação às necessidades latentes dos clientes. É natural que a gestão da qualidade tenha também acompanhado essa evolução. Ela deixou de ser direcionada principalmente para o chão da fábrica e passou a envolver todos os processos da organização. Assim, a gestão pela qualidade total tornou-se uma importante opção para as organizações conquistarem vantagem competitiva sobre os concorrentes.

Para Garvin (2002), quatro diferentes "eras da qualidade" podem ser definidas: a da inspeção; a do controle estatístico da qualidade; a da garantia da qualidade; e a da administração estratégica da qualidade.

De forma geral, de acordo com o que aponta Garvin (2002), embora tenha havido uma mudança contínua na forma pela qual se busca aumentar a qualidade nas empresas, a definição implícita do termo "qualidade" pouco se modificou ao longo das quatro "eras" citadas. A conformidade do produto em relação às especificações de projeto permaneceu como a definição mais utilizada na prática, apesar de, na teoria, ter havido grandes avanços.

Para Veronezi (2015), a adequação ao uso define o que se entende por qualidade. Ela está associada a um processo de melhoria contínua em produtos e serviços, o que não deixa de ser algo ligado à adequação ao uso realizado pelo cliente final. 
Garvin (2002) define a qualidade como a conformidade de produtos e serviços, com os requisitos do cliente final. Todas as definições estão voltadas para o uso que se faz de um produto ou serviço, direcionando o conceito de qualidade ao que é estabelecido pelo cliente final, que é o verdadeiro usuário.

Aparentemente, a evolução da gestão da qualidade apresentou praticamente as mesmas fases nos Estados Unidos. Contudo, o movimento de se voltar para o mercado e incorporar a qualidade na estratégia das empresas foi iniciado na década de 1980 nos Estados Unidos (GARVIN, 2002).

Diante disso, segundo Veronezi (2015), a garantia da qualidade do produto passou a ser de primordial importância para que se estabelecesse uma relação de confiança entre consumidor e produtor. Essa garantia está baseada em atividades que resguardam o consumidor de falhas no produto, constituindo o controle de qualidade de um produto ou serviço.

\section{PROCEDIMENTOS METODOLÓGICOS}

A pesquisa tem caráter qualitativo por se tratar do estudo das BPF (Boas Práticas de Fabricação), sendo ainda bibliográfica e documental, pois, para sua fundamentação, foram utilizados artigos, teses, livros, revistas e redes eletrônicas sobre os principais conceitos e práticas associados ao tema (COSTA, 2013).

\section{RESULTADOS E DISCUSSÃO}

Segundo Albuquerque (2013), no mercado de produtos perecíveis, a qualidade não é uma vantagem competitiva, mas sim, um requisito fundamental, ainda mais quando se refere a BPF. Em alguns casos é até mesmo requisito mandatório legal para poder comercializar os produtos, considerando-se que existem diversas formas de se atingir um alto padrão de qualidade. Uma das formas mais adotadas é a chamada BOAS PRÁTICAS DE FABRICAÇÃO - BPF-, a qual é composta por princípios e regras para o correto manuseio dos produtos, levando em conta desde as matérias-primas até o produto final. 


\subsection{A gestão da qualidade na indústria de alimentos}

Veronezi (2015) afirma que a qualidade deixou de ser um diferencial competitivo para transformar-se em uma condição para a permanência no mercado. Nesse contexto, qualidade passa a ser uma exigência absoluta dos consumidores e, portanto, dos mercados. As definições do termo qualidade sofreram mudanças consideráveis com o passar dos anos, passando de um simples conjunto de ações operacionais, centradas e localizadas em pequenas melhorias do processo produtivo, a um dos elementos fundamentais do gerenciamento das organizações, o que a torna fator crítico para a sobrevivência não só das empresas, mas também de produtos, processos e pessoas.

No segmento da indústria de alimentos, por exemplo, para melhor entendimento do conceito de qualidade, pode-se abordá-lo sob duas óticas, a saber: qualidade percebida e qualidade intrínseca.

A qualidade percebida está relacionada com as características do produto que levam à recompensa pelo seu consumo e que podem atrair o consumidor para a recompra. Dentre essas características, é possível citar a crocância, o odor, a cor, a textura, o aroma, enfim, as propriedades sensoriais em geral. Além dessas, contribuem para a qualidade percebida a composição nutricional e as características da embalagem.

Já a qualidade intrínseca é tudo aquilo que o consumidor considera como óbvio no produto, tais como peso correto do produto, ausência de contaminantes, não utilização de componentes proibidos pela legislação de dosagens seguras. Enfim, a qualidade intrínseca está relacionada com a segurança e o atendimento à legislação por parte do produto. Para o consumidor, um produto (ou serviço) é considerado de qualidade quando atende ao objetivo para o qual foi adquirido; para o produtor, a qualidade do produto corresponde à satisfação do consumidor (BERTOLINO, 2010).

Para Veronezi (2015), o grau de desenvolvimento do controle da qualidade é maior quando agregado a operações tais como: práticas de inspeção de campo e análises laboratoriais; investimentos na qualificação de equipes; e disposição de instrumental adequado para a melhoria do processo, desde a matéria-prima, insumos, serviços de água, materiais de embalagem, condições de higiene e sanitização, limpeza e a funcionalidade das instalações, condições de armazenamento e transporte, até o produto acabado. No setor alimentício, por exemplo, para a implantação de uma Gestão da Qualidade, é 
possível utilizar sistemas (métodos e procedimentos) já testados e literatura de fácil acesso. Trata-se dos Sistemas GMP (Guidelines for Development of Good Manufacturing Practices) e HACCP (Harzard Analysis and Critical Control Points), BPF (Boas Práticas de Fabricação) e APPCC (Análise de Perigos e Pontos Críticos de Controle).

\subsubsection{APPCC}

O sistema de análise de perigos e pontos críticos de controle (APPCC ou HACCP) é um método que tem por base a aplicação de princípios técnicos e científicos de prevenção, com a finalidade de garantir a inocuidade dos processos de produção, manipulação, transporte, distribuição e consumo dos alimentos, cobrindo todos os fatores que podem afetar sua segurança (VERONEZI, 2015).

No Brasil, a primeira legislação que remete ao APPCC surgiu em 1993, com procedimentos para o pescado. No mesmo ano, com a Portaria ${ }^{\circ} 1428$, do Ministério da Saúde, foram estabelecidas normas para a aplicação do APPCC em todas as indústrias de alimentos do Brasil. Em 1998, a Portaria n 46 do MAPA (Ministério da Agricultura, Pecuária e Abastecimento) obrigou que fosse realizada a implantação gradativa do programa de garantia de qualidade APPCC em todas as indústrias de produtos de origem animal, cujo pré-requisito essencial são as Boas Práticas De Fabricação (GARVIN, 2002).

De acordo com Veronezi (2015), a referida Portaria $\mathrm{n}^{\circ} 46$, de 10 de fevereiro de 1998, define APPCC como "um sistema de análise que identifica perigos específicos e medidas preventivas para seu controle, objetivando a segurança do alimento”.

\subsubsection{Boas Práticas de Fabricação}

De acordo com Veronezi (2015), as Boas Práticas de Fabricação são um conjunto de normas empregadas em produtos, processos, serviços e instalações que visam à promoção e certificação da qualidade e da segurança dos alimentos.

Rossiter (2008) define as BPF como o programa de segurança de alimentos que estabelece o alicerce do processo, descrevendo sua estrutura, procedimentos e organizações necessárias para garantir aspectos higiênico-sanitários na fabricação e 
manuseio de alimentos, tendo como principal objetivo garantir a integridade do alimento e a saúde do consumidor.

A ANVISA estabelece as BPF como sendo um conjunto de medidas que devem ser adotadas pelas indústrias de alimentos a fim de garantir a qualidade sanitária e a conformidade dos produtos alimentícios com os regulamentos técnicos. A legislação sanitária federal regulamenta essas medidas em caráter geral, as quais se aplicam a todo tipo de indústria de alimentos, e são voltadas às indústrias que processam determinadas categorias de alimentos (ANVISA, 2004).

As Boas Práticas consistem em um dos sistemas mais reconhecidos e de boa resposta para a obtenção de um alimento seguro, mantendo uma estreita relação com o consumidor, pois asseguram sua saúde, segurança e bem-estar. Elas promovem educação e qualificação nos aspectos de higiene, desinfecção e disciplina operacional. Assim, a segurança de alimentos é garantida com esforços combinados de todos os envolvidos na sua cadeia produtiva (VERONEZI, 2015).

\subsubsection{Implantação das boas práticas de fabricação}

De acordo com Veronezi (2015), a implantação das BPF em serviços de alimentação, além de ser uma exigência legal, eleva a qualidade dos produtos, protege a saúde pública, diminui gastos com internações hospitalares, dá maior segurança e satisfação ao comensal. Para a implantação dessas Boas Práticas, é necessário que se conheça o processo produtivo envolvido, bem como as limitações impostas pelo uso feito pelo cliente ou consumidor final, de forma a se ter uma visão sobre os perigos potenciais e os riscos de contaminação envolvidos. Com esses dados, é possível determinar o rigor e a profundidade das Boas Práticas de Fabricação a serem implementadas.

Para poder cobrar que as regras desse sistema sejam cumpridas pelos empregados, a empresa deve fornecer treinamento em manipulação de alimentos, incluindo programas de saúde e higiene pessoal a todos os novos colaboradores cujas atribuições estejam relacionadas com áreas de produção e controle de qualidade, sempre antes do início de suas atividades. O treinamento deve incluir também os colaboradores da área de manutenção e de outras áreas cuja atividade possa afetar a qualidade do produto. Periodicamente, e não excedendo o intervalo de um ano, os treinamentos devem ser 
reciclados e devidamente registrados. As indústrias de alimentos precisam descrever as operações realizadas em um manual, que consiste em um documento que inclua requisitos de manutenção preventiva e de higienização (instalações, dos equipamentos e dos utensílios), controle da água de abastecimento, controle integrado de vetores e pragas urbanas, controle da higiene e saúde dos manipuladores e o controle e garantia de qualidade do produto final (BERTOLINO, 2010).

\subsection{Procedimentos operacionais padronizados - POP}

De acordo com Martins (2013), Procedimento Operacional Padrão - em inglês Standard Operating Procedure (SOP)- consiste em descrever, em detalhes, todas as operações necessárias para realizar um determinado procedimento, ou seja, "um roteiro padronizado para realizar uma determinada atividade", sendo de grande importância dentro de qualquer processo funcional para garantir, mediante uma uniformização, os resultados desejados por cada tarefa realizada. Um exemplo de Procedimento Operacional Padrão no século passado pode ser encontrado nas linhas de montagem do modelo " $T$ " da Ford, onde todos os consumidores em potencial poderiam ter um automóvel desde que fosse da cor preta. Segundo a autora, no presente, podemos verificar que somente o carro preto não foi suficiente para satisfazer o consumidor, o que gerou a necessidade do desenvolvimento de novos modelos de Procedimentos Operacionais Padrão.

Para a ANVISA (2004), os POPs são procedimentos descritos de forma objetiva que definem as instruções para a realização de uma atividade na rotina da produção de alimentos, seja em sua elaboração, transporte ou armazenamento. Controle da potabilidade da água, manutenção preventiva e calibração de equipamentos, programa de recolhimento de alimentos, seleção de matérias-primas, ingredientes e embalagens, higienização das instalações, equipamentos, móveis e utensílios, manejo de resíduos e controle integrado de vetores e pragas urbanas são aspectos que requerem criação e manutenção de Procedimentos Operacionais Padronizados.

De acordo com Lima (2005), Procedimento Operacional Padrão é o documento que mostra o planejamento do trabalho com a sequência das atividades descritas detalhadamente, as quais devem ser executadas para atingir a meta padrão, sendo que este 
planejamento deve conter: listagem dos equipamentos; peças e materiais utilizados na tarefa, incluindo-se os instrumentos de medição; padrões da qualidade; descrição dos procedimentos da tarefa por atividades críticas; condições de fabricação, de operação e pontos proibidos de cada tarefa; pontos de controle (itens de controle e características da qualidade) e os métodos de controle; relação de anomalias passíveis de ação; roteiro de inspeção periódica dos equipamentos de produção. Para o autor, o POP tem como objetivo a busca da uniformização do processo ou atividade, ou seja, fazer que pessoas que executam a mesma tarefa possam realizá-las de forma invariável.

Já para Martins (2013), o objetivo do POP é sustentar o processo em funcionamento, por meio da padronização e da minimização de ocorrências de desvios na execução de atividades, ou seja, fazer que as ações tomadas para a garantia da qualidade sejam padronizadas. A elaboração de um POP deve seguir as seguintes etapas definidas: objetivos, descrição, monitoramento, ação corretiva, registros e verificação, podendo existir uma variação de acordo com a legislação a ser seguida. Os POPs devem ser aprovados, datados e assinados pelo responsável pelo estabelecimento.

\subsection{Legislação}

$\mathrm{O}$ art. $8^{\circ}$. da Lei Federal n. 9.782/99, criada pela ANVISA, incumbe a referida agência regulamentar, controlar e fiscalizar os produtos e serviços que envolvem risco à saúde pública. No parágrafo $1^{\circ}$., consideram-se bens e produtos submetidos ao controle e fiscalização sanitária pela agência: alimentos, inclusive bebidas; águas envasadas; seus insumos; suas embalagens; aditivos alimentares; limites de contaminantes orgânicos; e resíduos de agrotóxicos e de medicamentos veterinários (BRASIL, 1999).

A Resolução RDC $\mathrm{n}^{\circ} 275$, de 21 de outubro de 2002, foi desenvolvida com o propósito de atualizar a legislação geral, introduzindo o controle contínuo das Boas Práticas de Fabricação e os Procedimentos Operacionais Padronizados, além de promover a harmonização das ações de inspeção sanitária, por meio de instrumento genérico de verificação das Boas Práticas de Fabricação. Portanto, ela consiste em um ato normativo complementar à Portaria SVS/MS n 326/97 (BRASIL, 2002)

A resolução vigente e mais atual para o controle sanitário de alimentos no Brasil é a RDC $n^{\circ} 216 / 2004$, que estabelece procedimentos de boas práticas para serviços de 
alimentação, a fim de garantir as condições higiênico-sanitárias dos alimentos preparados (BRASIL, 2004).

\section{CONSIDERAÇÕES FINAIS}

O estudo sobre as Boas Práticas de Fabricação tem a intenção de demonstrar sua relevância quando bem implantadas de acordo com a realidade fabril, contribuindo não só na administração como também no desempenho de seus funcionários. Demonstra-se, assim, a importância de continuar realizando treinamento com os colaboradores sobre Boas Práticas de Fabricação, pois além de auxiliar em uma melhor produção, pode contribuir em todos os níveis operacionais da empresa, desenvolvendo colaboradores além de desempenhar suas funções, possam ter a garantia de qualidade e segurança no trabalho.

\section{REFERÊNCIAS}

ALBUQUERQUE, Daniela. Introdução às boas práticas de fabricação (BPF). 2013. Disponível em: <https://certificacaoiso.com.br/introducao-as-boas-praticas-defabricacao-bpf/>. Acesso em: 15 jul. 2019.

BERTOLINO, Marco Túlio. Gerenciamento da Qualidade na Indústria de Alimentos. São Paulo: Artmed, 2010. 320 p.

BRASIL.Ministério da Saúde. Agência Nacional de Vigilância Sanitária. ResoluçãoRDC $n^{\circ}$. 216, de 15 de setembro de 2004. Dispõe sobre o Regulamento Técnico de Boas práticas para serviços de alimentação. Diário Oficial da República Federativa do Brasil, Brasília, DF, 2004. Disponível em: < http://portal.anvisa.gov.br/documents/33916/388704/RESOLU\%25C3\%2587\%25C3\%2 $5830-$

RDC\%2BN\%2B216\%2BDE\%2B15\%2BDE\%2BSETEMBRO\%2BDE\%2B2004.pdf/2 3701496-925d-4d4d-99aa-9d479b316c4b >. Acesso em: 15 jul. 2019.

BRASIL. Agência Nacional de Vigilância Sanitária. Regularização de Empresas: Alimentos. 2004. Disponível em: <http://portal.anvisa.gov.br/registros-eautorizacoes/alimentos/empresas/boas-praticas-de-fabricacao>. Acesso em: 15 jul. 2019.

BRASIL. Ministério da Saúde. Agência Nacional de Vigilância Sanitária. ResoluçãoRDC $n^{\circ} 275$, de 21 de outubro de 2002. Dispõe sobre o Regulamento Técnico de Procedimentos Operacionais Padronizados aplicados aos Estabelecimentos Produtores/Industrializadores de Alimentos e a Lista de Verificação das Boas Práticas de Fabricação em Estabelecimentos Produtores/Industrializadores de Alimentos. Diário Oficial da República Federativa do Brasil, Brasília, DF, 2002. Disponível em: < 
http://portal.anvisa.gov.br/documents/10181/2718376/RDC_275_2002_COMP.pdf/fce9 dac0-ae57-4de2-8cf9-e286a383f254>. Acesso em: 15 jul. 2019.

BRASIL. Lei $\mathrm{n}^{\circ}$ 9.782, de 26 de janeiro de 1999. Diário Oficial da República Federativa do Brasil, Brasília, DF, 1999. Disponível em: < http://www.planalto.gov.br/ccivil_03/LEIS/L9782.htm >. Acesso em: 15 jul. 2019.

BRASIL.Ministério da Saúde. Secretaria de Vigilância Sanitária. Portaria n 326, de 30 de julho de 1999. Regulamento técnico sobre as condições higiênico-sanitárias e de boas práticas de fabricação para estabelecimentos produtores/industrializadores de alimentos. Brasília, Diário Oficial da República Federativa do Brasil, 1 ago. 1999 Disponível em: $<$ http://portal.anvisa.gov.br/documents/33916/388704/Portaria\%2BSVSMS\%2BN.\%2B326\%2Bde\%2B30\%2Bde\%2BJulho\%2Bde\%2B1997.pdf/87a1ab030650-4e67-9f31-59d8be3de167>. Acesso em: 15 jul. 2019.

COSTA, Mariana de Almeida. Gestão Estratégica: uma oportunidade para melhorar o resultado operacional. 2013. 104 f. Monografia (Especialização) - Curso de Engenharia de produção, Universidade Federal de Juiz de Fora, Juiz de Fora, 2013. Disponível em: <http://www.ufjf.br/ep/files/2014/07/2012_3_Mariana.pdf>. Acesso em: 04 jul. 2019.

GARVIN, D. A. Gerenciando a qualidade: a visão estratégica e competitiva. Rio de Janeiro: Qualitymark, 2002.

GENTA, T. M. S.; MAURICIO, A. A.; MATIOLI, G. Avaliação das Boas Práticas através de check-list aplicado em restaurantes self-service da região central de Maringá, Estado do Paraná. Acta Scientiarum. Health Sciences, Maringa, v. 27, n. 2, p. 151-156, 2005.

GIORDANO, J.C. Riscos à qualidade de alimentos e fármacos. Revista Controle de Contaminação, v. 6, n. 54, p. 22-25, 2003.

LIMA, R. "Procedimento Operacional Padrão" - A Importância de se padronizar tarefas nas BPLC. Curso de BPLC - Belém, 2005.

MARCHIORI, Cristiane. Diagnóstico e implantação de boas práticas de fabricação em uma indústria de conservas do município de Francisco Beltrão-PR. 2015. 65 f. Monografia (Especialização) - Curso de Gestão da Qualidade na Tecnologia de Alimentos, Universidade Tecnológica Federal do Paraná, Francisco Beltrão, 2015. Disponível em: <repositorio.roca.utfpr.edu.br/jspui/bitstream/1/7185/1/FB_GQTA_2014_05.pdf>. Acesso em: 15 jul. 2019.

MARTINS, R. Procedimento Operacional Padrão (POP). 2013.Disponível em: <http://www.blogdaqualidade.com.br/procedimento-operacional-padrao-pop/.> Acesso em: 15 jul. 2019. 
ROSSITER, K.W.L. Programa 5S: Alicerce para implantação de Sistemas e Boas Práticas de Fabricação na Indústria de Alimentos. Recife, Universidade Federal de Pernambuco: monografia de especialização, 2008. Disponível em:< http://www.repositorio.ufpe.br/bitstream/handle/123456789/5386/arquivo602_1.pdf?se qu ence $=1 \&$ isAllowed=y $>$. Acesso em: 15 jul. 2019.

SILVA, E.M. Implantação das Boas Práticas de Fabricação em uma agroindústria de produtos cárneos embutidos no município de São Jerônimo - RS. Trabalho de conclusão (Curso de Graduação Tecnológico em Planejamento e Gestão para o Desenvolvimento Rural). Universidade Federal do Rio Grande do Sul. Arroio dos Ratos, 2011. Disponível em: <https:/gepsaa.wordpress.com/2013/03/04/boas-praticas-defabricacao-em-agroindustrias/>. Acesso em: 15 jul. 2019.

VERONEZI, Camila. A importância da implantação das Boas Práticas de Fabricação na indústria de alimentos. Curitiba: Revista Saúde e Desenvolvimento, v. 8, n. 4, 2015. Semestral. Disponível em: <https://www.uninter.com/revistasaude/index.php/saudeDesenvolvimento/article/view/ 410>. Acesso em: 15 jul. 2019. 